\title{
Voxel Placement Precision for GABA-Edited Magnetic Resonance Spectroscopy
}

\author{
Xue Bai ${ }^{1}$, Ashley D. Harris ${ }^{2,3}$, Tao Gong, 2,4 , Nicolaas A. J. Puts ${ }^{2,3}$, Guangbin Wang4, \\ Michael Schär², Peter B. Barker ${ }^{2,3}$, Richard A. E. Edden ${ }^{2,3 *}$ \\ ${ }^{1}$ Department of Radiology, Qilu Hospital of Shandong University, Jinan, China \\ ${ }^{2}$ Russell H. Morgan Department of Radiology and Radiological Science, The Johns Hopkins University School of Medicine, \\ Baltimore, MD, USA \\ ${ }^{3}$ F. M. Kirby Research Center for Functional Brain Imaging, Kennedy Krieger Institute, Baltimore, MD, USA \\ ${ }^{4}$ Department of MR, Shandong Medical Imaging Research Institute, Shandong University, Jinan, China \\ Email: *raee2@jhu.edu
}

How to cite this paper: Bai, X., Harris, A.D., Gong, T., Puts, N.A.J., Wang, G.B., Schär, M., Barker, P.B. and Edden, R.A.E. (2017) Voxel Placement Precision for GABA-Edited Magnetic Resonance Spectroscopy. Open Journal of Radiology, 7, 3544.

https://doi.org/10.4236/ojrad.2017.71004

Received: February 22, 2017

Accepted: March 21, 2017

Published: March 24, 2017

Copyright ( $) 2017$ by authors and Scientific Research Publishing Inc. This work is licensed under the Creative Commons Attribution International License (CC BY 4.0).

http://creativecommons.org/licenses/by/4.0/ (c) (i) Open Access

\begin{abstract}
The purpose of the present study was to assess the reproducibility of voxel placement for GABA-edited MRS. GABA-edited MRS data were acquired in 13 healthy volunteers from $(3 \mathrm{~cm})^{3}$ voxel; and within the same session a second acquisition was independently prescribed. A three-dimensional voxel mask image was reconstructed in T1-image-space using the SVMask tool (in house software). Reproducibility of voxel placement was assessed using the Dice overlap coefficient, both within-subject and between-subject following co-registration of $\mathrm{T} 1$ images and transformation of voxel mask images to standard space. Within-subject overlap coefficients were $86 \% \pm 5 \%$. Betweensubject overlap coefficients were $75 \% \pm 10 \%$. For the two voxel locations considered (occipital and sensorimotor), voxel overlap was very similar. Betweensubject values are higher due to between-session effects, anatomical variability and volume mismatch in standard space. While surprisingly low in terms of volume overlap, the overlap coefficients correspond to acceptable linear displacements.
\end{abstract}

\section{Keywords}

MRS, Voxel Placement, Precision, Voxel Prescription, Registration

\section{Introduction}

$1 \mathrm{H}$ magnetic resonance spectroscopy (MRS) is a non-invasive technique that allows for the quantitative investigation of a number of in vivo metabolites, such as $\mathrm{N}$-acetyl aspartate, creatine and choline, commonly interpreted as markers for 
neuronal integrity, energetic status and membrane turnover respectively [1]. Recently, there has been significant interest in using edited MRS to reveal signals from less concentrated metabolites, such as GABA [2] [3], glutathione [4] and ascorbate [5]. Due to the fundamental insensitivity of MRS, larger measurement volumes are required for these edited experiments, typically on the order of ( 3 $\mathrm{cm})^{3}$ compared to $(2 \mathrm{~cm})^{3}$ or less for traditional single-voxel MRS [6].

In cohort MRI studies, one common post-processing step is the co-registration of images either to each other, or to a standard-space template. Co-registration of images across a cohort allows comparisons between images to be drawn for anatomically equivalent regions, limited only by the quality of co-registration. In contrast, MRS is usually performed as a single-voxel measurement, and placement of voxels involves planning on the basis of predefined anatomical or functional landmarks. This is an irreversible anatomical judgment that cannot be mitigated by post-processing co-registration. For single-voxel acquisitions, the measurement acquired corresponds to the region prescribed without any further spatial resolution, or opportunity for spatial realignment. Conclusions drawn from MRS studies are usually based upon the functional role of a particular anatomical structure, and there is generally an implicit assumption that voxel placement is accurate and reproducible. To date, a landmark-based approach is the main method for edited MRS voxel placement. Given the reliance on anatomical landmarks, it is therefore particularly important to assess the reproducibility of voxel placement in MRS studies [7], especially for edited MRS experiments.

To our knowledge, no previous study has investigated the placement reliability of voxel for edited MRS, compared with traditional MRS. The increase in voxel size required for edited MRS of less concentrated metabolites may have several impacts on placement precision: displacement of the voxel by a fixed distance will have a relatively smaller impact on the voxel contents; larger voxels may suffer from additional anatomical restrictions; and operator care or judgment may be influenced by the size of the voxel to be planned. One further development that is relevant for studies of GABA is the increasing adoption of functionally motivated measurement regions, as opposed to anatomically defined studies. Tolerance of placement variance might be greater for a fronto-parietal region than a primary sensorimotor region, both because of the greater specificity of the functional definition and the qualitative difference in the conclusions likely to be drawn from such a study.

In this current study, the reproducibility of voxel placement for two anatomically defined and functionally motivated, regions of interest (occipital and sensorimotor), commonly used in MRS studies of sensory and motor function [8] [9] [10] [11], was assessed. Using the Dice overlap coefficient (DOC), placement precision both within-subject within-session, and between-subject was investigated. Differences between within- and between-subject results are discussed in terms of the interpretation of individually different anatomy and the limitations of the DOC. 


\section{Methods}

\subsection{Participants}

13 healthy male subjects (all right handed, age $30 \pm 6.1$ years old) participated in the study. Only male participants were included to mitigate gender effects on brain anatomy and voxel localization. Written informed consent was obtained for each participant under the approval of the local Institutional Review Board prior to testing.

\subsection{Edited-MRS}

Data were acquired on a Philips 3T "Achieva" MRI scanner (Best, the Netherlands) using a 32-channel head coil for receive and body coil for transmit. For each participant, sagittal $1 \mathrm{~mm}^{3}$ isotropic T1-weighted (T1w) images (MPRAGE) were acquired and resliced in axial and coronal views (TR $=7.99 \mathrm{~ms}$, TE $=3.76 \mathrm{~ms}$, Flip angle $\left.=8^{\circ}\right)$. GABA-edited MRS voxels were manually placed in two regions (with the visualized voxel in Figure 1 correct for the 3 ppm GABA signal) viewing all 3 planes by a single experimenter. A $(3 \mathrm{~cm})^{3}$ voxel was placed on the right sensorimotor cortex (SM1, Figure 1(b)) and was centered on the central sulcus posterior to the hand-knob [12] in the axial plane; the voxel was rotated to align with the cortical surface by rotating in the coronal plane and subsequently in the sagittal plane. A second $(3 \mathrm{~cm})^{3}$ voxel was placed in the occipital cortex (OCC, Figure 1(c)), centered on the midline and rotated in the sagittal slice to align along the cerebellar tentorium and placed as posterior as possible without including the sagittal sinus or skull. Each voxel was placed twice in all participants. The first placement was part of a standard GABA-edited MEGA-PRESS scan with the following scan parameters: TE/TR $=68 / 2000 \mathrm{~ms}$, 320 transients acquired with editing pulses placed at 1.9 (edit-ON) and 7.5 (edit-OFF) ppm, $2 \mathrm{k}$ bandwidth and VAPOR water suppression(as described in [8]). The second placement was only performed to log voxel location parameters, although minimal MRS data were acquired (a 12-second water acquisition). Prior to the second placement, voxel location and angulations were zeroed so that the voxel was centered approximately in the center of the brain without rotation, and independent placement was again performed on the basis of the landmarks described above. All voxel placements were performed by a single experimenter and participants were not removed from the scanner in between the first and second voxel placement. No additional information (e.g. screen shots of the first placement) was used for the second placement. A total of 51 MRS voxels' data, from 13 participants were included in this study (1 subject's second SM1 voxel was unavailable).

\subsection{Analysis}

The following image analysis pipeline was used (Figure 2):

1) Generation of the MRS voxel mask (Figure 2(a)). Each MRS acquisition volume was reconstructed as a binary mask in the image matrix of the T1w image of the same subject using the SVMask tool (in house software), which ex- 
tracts the required geometric information from MRS and MRI file headers.

2) Brain extraction (Figure 2(b)). Skull-stripping of 3D T1w images was performed using the Brain Extraction Tool (BET, v2.1) [13], from the FSL suite.

3) Image co-registration (Figure 2(b)). T1w images were co-registered to (2 $\mathrm{mm})^{3}$ MNI standard-space brain using FMRIB's Linear Image Registration Tool (FLIRT, v6.0) [14].

4) Voxel transformation to standard space (Figure 2(c)). For each subject, the transformation matrix determined in step 3 was applied to all the voxel masks generated in step 1 to give voxel masks in standard space (as shown in Figure 1).

\subsubsection{Within-Subject Overlap}

The quantification of voxel overlap within subjects between the two scans was performed using the Dice overlap coefficient (DOC [15]). The DOC is defined as the intersection volume, divided by the mean volume of the two voxels; it ranges between 0 and 1, where 1 represents perfect overlap. For example, A might refer to the first OCC voxel mask and B to the second OCC voxel mask:

$$
\mathrm{DOC}=\frac{2(A \cap B)}{A+B} .
$$

Within-subject voxel overlap DOC was calculated using FSL tools in subjectspace (rather than standard space), prior to step 2 above (as shown in Figure 2(a)).

\subsubsection{Between-Subject Overlap in Standard Space}

Between-subject voxel placement reliability was calculated using the firstplacement voxel masks for each region from the 13 participants, registered to standard space. For each of the thirteen subjects, another subject was selected randomly (without replacement and prohibiting double comparisons e.g. 1-8 and 8-1) to generate thirteen unique pair-wise comparisons. The DOC was calculated for OCC and SM for each of these pairs. This process was repeated five times, so that in all 65 between-subject overlap coefficients were calculated for each region.

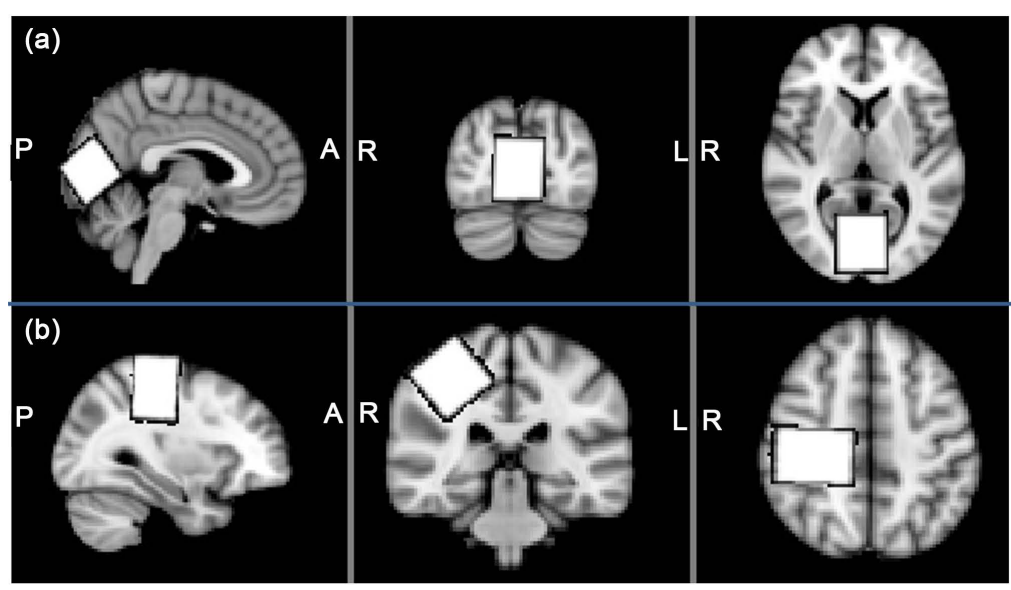

Figure 1. MRS voxel placement. A single-subject voxel, shown in white, is superimposed on the average template brain, in standard space. Both occipital (a) and sensorimotor (b) regions are considered. 


\subsubsection{Voxel Density Images}

In standard space, an image was calculated of the sum of the voxel masks of all subjects (separately for OCC and SM voxel). For each point in space, this image reflects how often that point is included in the different subjects' MRS voxels.

\section{Results}

\subsection{Within-Subject Voxel Overlap}

The overlap between the first and second voxel prescriptions for each subject were $87 \% \pm 5 \%$ in the occipital region and $86 \% \pm 5 \%$ in the SM region (Figure $3)$. The displacement between the centers of the two voxels was $2.6 \pm 1.2 \mathrm{~mm}$ (mean \pm standard deviation), with very similar average displacements for both the OCC and SM locations (2.66 $\mathrm{mm}$ for SM and $2.63 \mathrm{~mm}$ for OCC).

\subsection{Between-Subject Voxel Overlap}

Mean between-subject voxel overlap was $75 \% \pm 10 \%$ in OCC and $78 \% \pm 7 \%$ in SM (as shown in Figure 3 and Figure 4). Due to substantial variation in brain volume between subjects (from 1.05 liter to 1.50 liter for males [16]), the volume of the MRS voxels is scaled in standard space through the registration process. Voxel volumes were scaled relative to the mean by $-13 \%$ to $+15 \%$ (standard deviation 9\%). The mean pair-wise volume mismatch is $11 \%$.

(a)

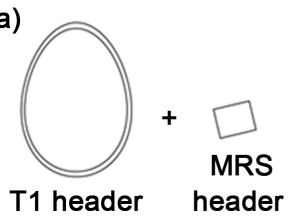

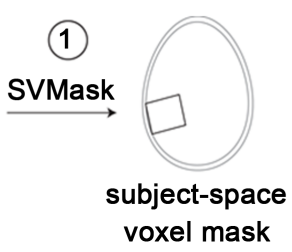

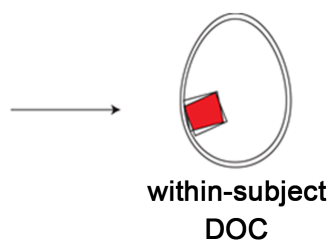

DOC (b)

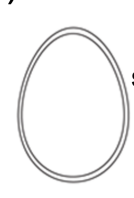

T1W image
(2)

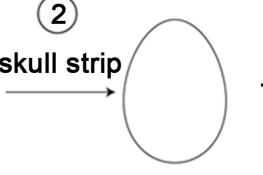

(c)

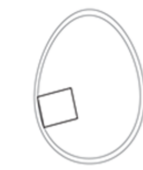

subject-space voxel mask

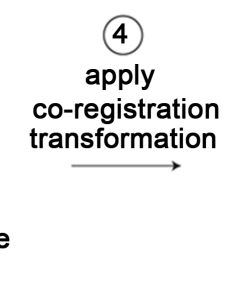

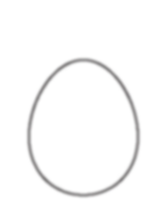

template

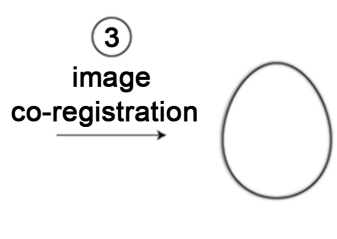

Figure 2. Schematic outline of the registration framework. Numbered steps correspond to the list in Analysis Methods above. (a) Voxel header information is used to reconstruct a voxel mask image (step 1). Masks from the two voxel placements (per region) are then compared to give the within-subject DOC; (b) T1W images are skull stripped (step 2) and co-registered to a standard-space template (step 3); (c) The co-registration transformation is applied to voxel masks (step 4), to allow between-subject DOC comparisons.

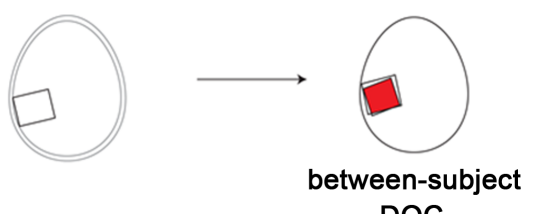

DOC 


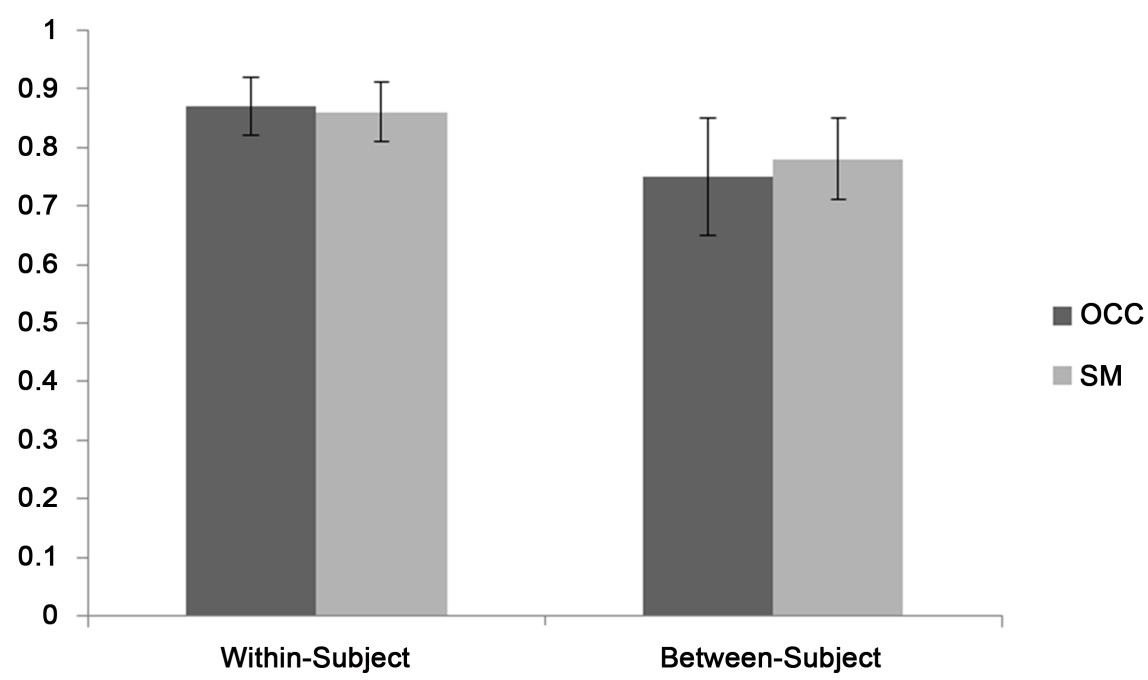

Figure 3. Overlap coefficients (DOC) calculations of MRS voxels within- and betweensubjects. $\mathrm{OCC}=$ occipital; $\mathrm{SM}=$ sensorimotor.

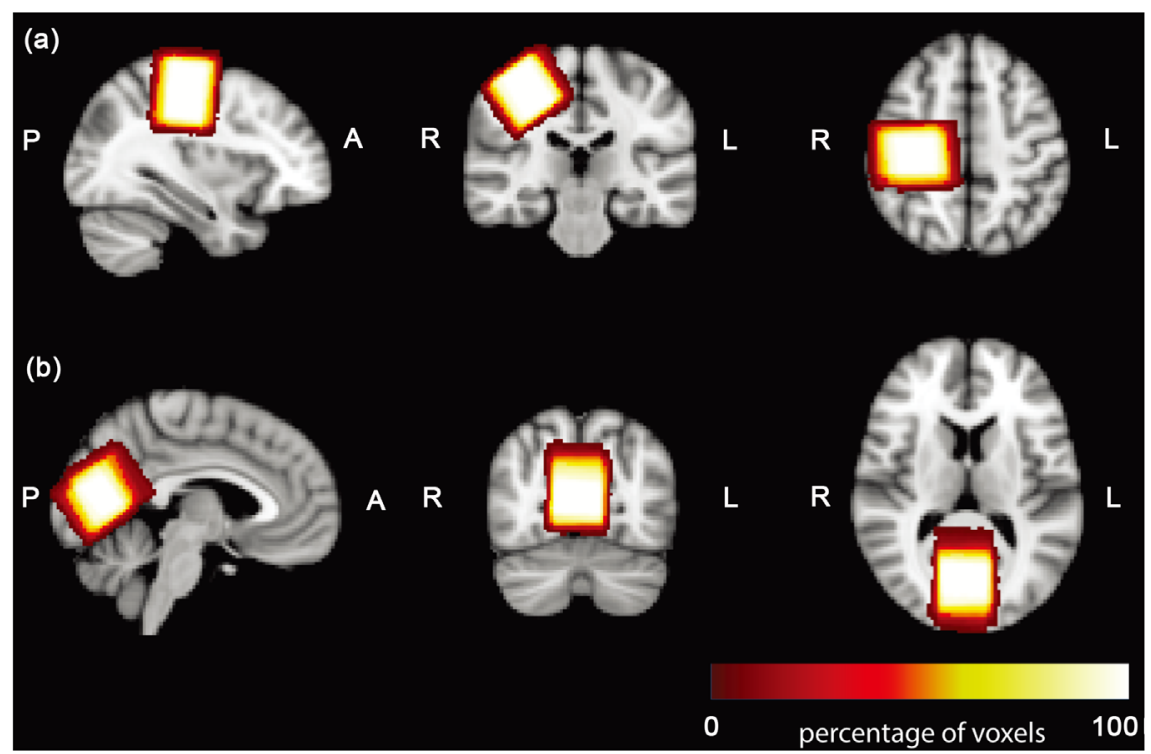

Figure 4. Voxel density images. All the OCC (a) and SM (b) voxels are transferred to one standard space. The degree of spatial overlap illustrates the repeatability of MRS voxel placement procedures.

\section{Discussion}

It is a tacit assumption of the majority of single-voxel MRS studies that metabolite concentrations are measured from equivalent regions in each subject. It is therefore somewhat surprising that the fidelity of voxel placement has only occasionally been investigated in the literature [7] [17]. In order to evaluate the repeatability of voxel placement for MEGA-PRESS GABA scans, this study calculated the DOC both within-subject and, after image co-registration, betweensubject. The primary results suggest the within-subject DOC is $85 \%$ in both occipital and sensorimotor measurement regions, and that the between subject DOC is $75 \%$ for a $3 \times 3 \times 3 \mathrm{~cm}^{3}$ voxel. 
At first glance, these overlap numbers are surprisingly low; however, $85 \%$ overlap is equivalent to $5 \%$ (or $1.5 \mathrm{~mm}$ of one direction of a $(3 \mathrm{~cm})^{3}$ voxel) displacements along each of the three spatial directions, without any variability in rotations. The mean displacement between voxel centers is $2.6 \mathrm{~mm}$, equal to the diagonal of a $1.5 \mathrm{~mm}$ cube, suggesting that displacement accounts for the majority of the overlap loss, with only minor losses due to rotation. Given that voxels are placed using $\mathrm{T} 1 \mathrm{w}$ images with 1-mm-isotropic resolution, precision better than $1 \mathrm{~mm}$ in each direction would not be expected. Similarly, the $75 \%$ overlap between subjects corresponds to a $9 \%$ (or $2.7 \mathrm{~mm}$ ) displacement along all three axes-again not substantially greater than the $(2 \mathrm{~mm})^{3}$ matrix on which co-registration was performed.

Some limitations arise from the choice of the Dice coefficient (DOC). Firstly, the DOC only reports on the overlap between the tissues contained in different voxels, and cannot address the impact that any change in voxel contents has on measured GABA concentration. Secondly, it is difficult to interpret the difference in DOC from within-to between-subjects comparisons (85\% vs. $75 \%$ ). Some of this reduction in overlap is "real", reflecting the operator's variable interpretation of individual anatomy, while some of it is artifactual reflecting imperfections in co-registration on a $(2 \mathrm{~mm})^{3}$ matrix.

A further limitation of using the Dice coefficient in standard space is that two voxels with identical position and orientation that originate from different-sized brains will not give overlap of $100 \%$ due to a volume mismatch in standard space. In this special case, the Dice coefficient is less than 1 by half the fractional difference in volume between the brains. This suggests that the mean volume mismatch in our cohort (11\%) therefore accounts for about half of the additional between-subject overlap loss compared to within-subject. Thus, while the DOC reflects the mathematical overlap between voxels, in standard space it does not report simply on operator reliability. One might even suggest that voxel volume should be scaled relative to total brain volume when MRS scans are prescribed (which would likely result in increased DOC), but this has signal-to-noise (SNR) and data quality implications also. Additional limitations are the consideration of only two voxel positions, the lack of within-subject between-session data, and the single operator prescribing voxels. Although the strong agreement between the two regions studied suggests that the findings may be generalizable, these results are likely to be affected to some degree by several factors including the complexity of the prescription protocol including the number of rotations, reproducibility of subject placement in the scanner (i.e. brain orientation in the anatomical images), and ease of identification of landmarks used (which may differ due to lesions, atrophy or normal/abnormal anatomical variation). Additional advances in co-registration, as well as an in-depth investigation of the effect of small changes in voxel tissue composition on GABA levels, would allow for a better understanding of the effect of small changes in voxel placement within and between subjects. Furthermore, we restricted our investigation to single-prescriber, single-scanner and single-session. For longitudinal or mul- 
ti-center studies, the effects of multi-prescriber, multi-scanner, and multi-session on voxel localization and overlap would need to be investigated as well.

In practical terms, this study shows that voxel placement to a precision of 2 - 3 $\mathrm{mm}$ in three directions is possible, with care. Although these results give surprisingly low Dice coefficients of $\sim 75 \%$, this level of precision is approximately equal to the ability of subjects to remain motionless during the 10-minute scan. This agreement is only possible due to the rigorous specification of voxel placement protocol, including three-dimensional position and rotation information. Of particular note is the need to specify the order of multiple voxel rotations (which do not commute) for voxels. A range of voxel overlaps have been shown previously, from $57 \%$ within-subject for a small $(15 \mathrm{~mm})^{3}$ parietal voxel [7] to $86 \%$ for a slightly larger $(20 \mathrm{~mm})^{3}$ posterior cingulate voxel [17]. There is some evidence that automated voxel placement protocols, which typically calculate voxel location parameters from parameters co-registering images to standard space within-session, can perform as well as human operators and remove some variance [17], and prospective voxel placement correction has been demonstrated with a navigator-based acquisition [18]. Another methodological improvement is the use of voxel density maps (as used in Gaetz et al. [19]) to simultaneously display the position of MRS voxels and the placement precision across subjects.

\section{Conclusion}

In conclusion, the percentage of equivalent tissue included by $3 \times 3 \times 3 \mathrm{~cm}^{3}$ MRS voxels in different subjects is surprisingly low at $75 \%$. However, this corresponds to displacements of less than $3 \mathrm{~mm}$ along three axes, which seems to be relatively good agreement, and some fraction of this overlap loss is caused by brain volume mismatches. Within-subject agreement is $\sim 85 \%$, again low at first glance, but equivalent to acceptable 3D displacements of $1.5 \mathrm{~mm}$ in all three directions.

\section{Acknowledgements}

This work was supported by NIH grants R01 EB016089 and P41 EB015909.

\section{References}

[1] Rae, C.D. (2014) A Guide to the Metabolic Pathways and Function of Metabolites Observed in Human Brain $1 \mathrm{H}$ Magnetic Resonance Spectra. Neurochemical Research, 39, 1-36. https://doi.org/10.1007/s11064-013-1199-5

[2] Mullins, P.G., McGonigle, D.J., O’Gorman, R.L., Puts, N.A., Vidyasagar, R., Evans, C.J. and Edden, R.A. (2014) Current Practice in the Use of MEGA-PRESS Spectroscopy for the Detection of GABA. NeuroImage, 86, 43-52.

[3] Rothman, D.L., Petroff, O.A., Behar, K.L. and Mattson, R.H. (1993) Localized 1H NMR Measurements of Gamma-Aminobutyric Acid in Human Brain in Vivo. Proceedings of the National Academy of Sciences of the United States of America, 90, 5662-5666. https://doi.org/10.1073/pnas.90.12.5662

[4] Terpstra, M., Henry, P.G. and Gruetter, R. (2003) Measurement of Reduced Gluta- 
thione (GSH) in Human Brain Using LCModel Analysis of Difference-Edited Spectra. Magnetic Resonance in Medicine, 50, 19-23. https://doi.org/10.1002/mrm.10499

[5] Terpstra, M., Marjanska, M., Henry, P.G., Tkac, I. and Gruetter, R. (2006) Detection of an Antioxidant Profile in the Human Brain in Vivo via Double Editing with MEGA-PRESS. Magnetic Resonance in Medicine, 56, 1192-1199. https://doi.org/10.1002/mrm.21086

[6] Puts, N.A.J. and Edden, R.A.E. (2012) In Vivo Magnetic Resonance Spectroscopy of GABA: A Methodological Review. Progress in Nuclear Magnetic Resonance Spectroscopy, 60, 29-41. https://doi.org/10.1016/j.pnmrs.2011.06.001

[7] Lee, H., Caparelli, E., Li, H., Mandal, A., Smith, S.D., Zhang, S., Bilfinger, T.V. and Benveniste, H. (2013) Computerized MRS Voxel Registration and Partial Volume Effects in Single Voxel ${ }^{1}$ H-MRS. Magnetic Resonance Imaging, 31, 1197-1205. https://doi.org/10.1016/j.mri.2013.04.001

[8] Edden, R.A., Crocetti, D., Zhu, H., Gilbert, D.L. and Mostofsky, S.H. (2012) Reduced GABA Concentration in Attention-Deficit/Hyperactivity Disorder. Archives of General Psychiatry, 69, 750-753.

https://doi.org/10.1001/archgenpsychiatry.2011.2280

[9] Puts, N.A., Edden, R.A., Evans, C.J., McGlone, F. and McGonigle, D.J. (2011) Regionally Specific Human GABA Concentration Correlates with Tactile Discrimination Thresholds. Journal of Neuroscience, 31, 16556-16560. https://doi.org/10.1523/JNEUROSCI.4489-11.2011

[10] Stagg, C.J., Bachtiar, V. and Johansen-Berg, H. (2011) The Role of GABA in Human Motor Learning. Current Biology, 21, 480-484. https://doi.org/10.1016/j.cub.2011.01.069

[11] Violante, I.R., Ribeiro, M.J., Edden, R.A., Guimaraes, P., Bernardino, I., Rebola, J., Cunha, G., Silva, E. and Castelo-Branco, M. (2013) GABA Deficit in the Visual Cortex of Patients with Neurofibromatosis Type 1: Genotype-Phenotype Correlations and Functional Impact. Brain, 136, 918-925. https://doi.org/10.1093/brain/aws368

[12] Yousry, T.A., Schmid, U.D., Alkadhi, H., Schmidt, D., Peraud, A., Buettner, A. and Winkler, P. (1997) Localization of the Motor Hand Area to a Knob on the Precentral Gyrus. A New Landmark. Brain, 120, 141-157. https://doi.org/10.1093/brain/120.1.141

[13] Smith, S.M. (2002) Fast Robust Automated Brain Extraction. Human Brain Mapping, 17, 143-155. https://doi.org/10.1002/hbm.10062

[14] Jenkinson, M. and Smith, S. (2001) A Global Optimisation Method for Robust Affine Registration of Brain Images. Medical Image Analysis, 5, 143-156. https://doi.org/10.1016/S1361-8415(01)00036-6

[15] Dice, L.R. (1945) Measures of the Amount of Ecologic Association between Species. Ecology, 26, 297-302. https://doi.org/10.2307/1932409

[16] Cosgrove, K.P., Mazure, C.M. and Staley, J.K. (2007) Evolving Knowledge of Sex Differences in Brain Structure, Function, and Chemistry. Biological Psychiatry, 62, 847-855. https://doi.org/10.1016/j.biopsych.2007.03.001

[17] Hancu, I., Blezek, D.J. and Dumoulin, M.C. (2005) Automatic Repositioning of Single Voxels in Longitudinal ${ }^{1} \mathrm{H}$ MRS Studies. NMR in Biomedicine, 18, 352-361. https://doi.org/10.1002/nbm.965

[18] Keating, B., Deng, W., Roddey, J.C., White, N., Dale, A., Stenger, V.A. and Ernst, T. (2010) Prospective Motion Correction for Single-Voxel 1H MR Spectroscopy. Magnetic Resonance in Medicine, 64, 672-679. https://doi.org/10.1002/mrm.22448 
[19] Gaetz, W., Bloy, L., Wang, D.J., Port, R.G., Blaskey, L., Levy, S.E. and Roberts, T.P. (2014) GABA Estimation in the Brains of Children on the Autism Spectrum: Measurement Precision and Regional Cortical Variation. NeuroImage, 86, 1-9.

Submit or recommend next manuscript to SCIRP and we will provide best service for you:

Accepting pre-submission inquiries through Email, Facebook, LinkedIn, Twitter, etc. A wide selection of journals (inclusive of 9 subjects, more than 200 journals)

Providing 24-hour high-quality service

User-friendly online submission system

Fair and swift peer-review system

Efficient typesetting and proofreading procedure

Display of the result of downloads and visits, as well as the number of cited articles

Maximum dissemination of your research work

Submit your manuscript at: http://papersubmission.scirp.org/

Or contact ojrad@scirp.org 